\title{
Determinación de perfiles plasmídicos de Escherichia coli y Klebsiella pneumoniae productoras de ß-lactamasas de espectro extendido aisladas en urocultivos
}

\section{Plasmid profile determination of Escherichia coli and Klebsiella pneumoniae producers of extended spectrum ß-lactamase isolated in urocultives}

\author{
Carlos Suarez ${ }^{1, a}$, Mario Monteghirfo ${ }^{2, b}$, Edgar Gonzales ${ }^{3, c}$ \\ ${ }^{1}$ Hospital Nacional Dos de Mayo. Lima, Perú \\ ${ }^{2}$ Facultad de Medicina, Universidad Nacional Mayor de San Marcos. Lima, Perú \\ ${ }^{3}$ Instituto Nacional de Salud del Niño. Lima, Perú \\ ${ }^{a}$ Licenciado Tecnólogo Médico, ${ }^{\mathrm{b}}$ Doctor en Ciencias Biológicas, ${ }^{\mathrm{C}}$ Magister en Microbiología \\ Tesis presentada para optar el Título Profesional de Licenciado en Tecnología Médica en el Área de Laboratorio Clínico y Anatomía Patológica de la Facultad de Medicina de \\ la Universidad Nacional Mayor de San Marcos.
}

An Fac med. 2018;79(1):35-43 / http://dx.doi.org/10.15381/anales.v79i1.14590

\begin{abstract}
Correspondencia:
Carlos Suarez Rojas

karloz_0891@hotmail.com

Av. Chimpu Ocllo 261 Urb. Santa Isabel-

Carabayllo.

Teléfonos: 945434400
\end{abstract}

Recibido: 23 enero 2018

Aceptado: 24 abril 2018

Conflictos de interés: Ninguno.

Fuentes de financiamiento:

Autofinanciado.

Citar como: Suarez C, Monteghirfo M, Gonzales E. Determinación de perfiles plasmídicos de Escherichia coli y Klebsiella pneumoniae productoras de B-lactamasas de espectro extendido aisladas en urocultivos. An Fac med. 2018;79(1):35-43

DOI: http://dx.doi.org/10.15381/anales. v79i1.14590

\section{Resumen}

Introducción. El aumento de las tasas de resistencia en bacterias uropatógenas y su propagación se han convertido en un problema de salud pública a nivel mundial, cobrando mayor importancia la diseminación de aislamientos resistentes productores de ß-lactamasas de espectro extendido, resultando útil la caracterización molecular de plásmidos y otros elementos genéticos de las bacterias para establecer la relación genética que existe entre ellas y tener datos relevantes que puedan sugerir la dirección de propagación de este tipo de resistencia. El objetivo de nuestra investigación fue determinar los perfiles plasmídicos en aislamientos de Escherichia coli y Klebsiella pneumoniae productoras de ß-lactamasas de espectro extendido de urocultivos del Instituto Nacional de Salud del Niño, Lima, Perú. Métodos. Estudio descriptivo, observacional de corte transversal. Resultados. Se determinaron diferentes patrones electroforéticos, obteniéndose de 1 hasta 7 bandas por muestra y en total 15 bandas de distinto tamaño, variando desde $1.5 \mathrm{~kb}$ hasta más de $20 \mathrm{~kb}$. Teniendo en cuenta los patrones de huella obtenidos, los aislamientos estudiados se distribuyeron en diferentes grupos o ramas, usando el programa NTSYSpc 2.1 y el algoritmo de agrupamiento UPGMA. Conclusión. No se encontró similitud genética marcada entre los aislamientos de origen comunitario y de origen asociado a atención sanitaria, por lo que no se puede establecer una relación significativa y determinar un origen común entre los mismos.

Palabras clave: Bacterias; beta-Lactamasas; Plásmidos; Escherichia coli; Klebsiella pneumoniae (fuente: DeCS BIREME).

\section{Abstract}

Introduction. The increase in resistance rates in uropathogenic bacteria and their spread has become in a significant worldwide public health, becoming more important the spread of resistant isolates producing of extended spectrum ß-lactamases, which are useful the molecular characterization of plasmids and other genetic elements of the bacteria to establish the genetic relationship between them, to have relevant data that we can suggest the direction of propagation of this type of resistance. The object of research was to determine the plasmid profiles in isolates of Escherichia coli and Klebsiella pneumoniae producing of extended spectrum ß-lactamases from urine cultures of the National Institute of Child Health, Lima, Peru. Methods. Descriptive, observational cross-sectional study. Results. Were detected different electrophoretic patternsin the samples, obtained from 1-7 bands per sample and a total of 15 bands of different sizes, which varied from $1.5 \mathrm{~kb}$ to more than $20 \mathrm{~kb}$. Considering the fingerprint patterns, the isolates analyzed were distributed into different clusters or branches, using the NTSYSpc 2.1 program and the UPGMA clustering algorithm. Conclusions. No genetic similarity was found between isolates of community origin and of origin associated with health care, so it is not possible to establish a meaningful relationship and to determine a common origin among them. Keywords: Bacteria; beta-Lactamases; Plasmids; Escherichia coli; Klebsiella pneumoniae (source: MeSH NLM). 


\section{INTRODUCCIÓN}

La resistencia a los antibióticos constituye un problema serio de salud pública en el mundo, el cual se ha agudizado durante los últimos años, porque a pesar de disponer nuevos antibióticos, el ritmo del desarrollo de la resistencia bacteriana en los diferentes patógenos representa un constante desafío terapéutico ${ }^{(1)}$.

La resistencia a antibióticos puede atribuirse a diferentes mecanismos; sin embargo, el mecanismo más frecuente e importante desde el punto de vista terapéutico en bacilos Gram negativos es la producción de enzimas tipo $\beta$-lactamasas. Las infecciones causadas por microorganismos productores de $\beta$-lactamasas de espectro extendido (BLEE) son difíciles de manejar. Desde su primer reporte en Alemania en 1983, la tasa de aislamientos de bacterias productoras de ß-lactamasas de espectro extendido en infecciones urinarias, especialmente Escherichia coli y Klebsiella pneumoniae, ha ido en aumento y se ha convertido en un gran problema a nivel mundial. En primer lugar, la terapia empírica a menudo fracasa, en segundo lugar, estos organismos tienden a ser resistentes a otros antimicrobianos incluyendo las fluoroquinolonas y aminoglucósidos $(2,3,4)$.

La selección de aislados bacterianos portadores de BLEE ha dificultado enormemente el tratamiento antibiótico de numerosas infecciones bacterianas porque presentan resistencia a la gran mayoría de los $\beta$-lactámicos y altas tasas de resistencias a los antimicrobianos de otras familias. Estas bacterias resistentes pueden transmitir los genes de resistencia por transferencia horizontal, mediante intercambio de plásmidos, generando así un problema terapéutico $(2,5,6,7)$.

Es importante tener un mejor conocimiento de los elementos génicos móviles que facilitan la difusión de los genes bla y otros genes de resistencia asociados, porque se agrava la resistencia cuando ocurre la transmisión de genes que codifican estas enzimas a otras enterobacterias ${ }^{(8)}$.

El objetivo del presente estudio fue determinar los patrones de resistencia y los perfiles plasmídicos de aislamientos de Escherichia coli y Klebsiella pneumo- niae, teniendo como finalidad determinar si se trata de una situación producida por una misma cepa o si, por el contrario, se tratan de cepas similares de una misma especie pero no idénticas; por lo que fue necesario estudiar la identidad o no entre cepas aisladas de pacientes enfermos hospitalizados y del sitio que se sospecha pueda ser el foco de contaminación (aislamientos de la comunidad). Existen varias técnicas de biología molecular, dentro de las cuales se encuentra el estudio de los perfiles plasmídicos, con la finalidad de establecer la relación epidemiológica existente y de esta manera obtener datos relevantes que nos puedan sugerir la dirección de propagación de este tipo de resistencia y aplicar las medidas control necesarias según sea el caso.

\section{MÉTODOS}

Se obtuvieron aislamientos bacterianos de Escherichia coli y Klebsiella pneumoniae en urocultivos de pacientes hospitalizados recibidos durante los meses de noviembre y diciembre del año 2012, del Servicio de Microbiología del Instituto Nacional del Niño, Lima, Perú. En total se obtuvieron 280 aislamientos positivos para o Klebsiella pneumoniae, de los cuales 62 aislamientos fueron de Escherichia coli y Klebsiella pneumoniae productoras de ß-lactamasas de espectro extendido

Los criterios de inclusión fueron: aislamientos de Escherichia coli y Klebsiella pneumoniae con resultado positivo para la detección de BLEE mediante el método de Jarlier - sinergia de discos (Comité de la Sociedad Francesa de Microbiología), y aislamientos de Escherichia coli y KlebsieIla pneumoniae obtenidos en pacientes entre 0 y 14 años de edad.

Adicionalmente se realizaron aislamientos procedentes de comunidad. Se consideraron aquellos obtenidos en pacientes que acudían por consulta externa o quienes tuvieron ingreso por emergencia con menos de 48 horas de hospitalización, también en el Instituto Nacional del Niño en el mismo tiempo de estudio.

El aislamiento bacteriano y las pruebas de susceptibilidad se realizaron en el Laboratorio de Microbiología del Intituto
Nacional de Salud del Niño previa autorización según protocolo de las jefaturas correspondientes. Los aislamientos de Escherichia coli y Klebsiella pneumoniae productoras de ß-lactamasas de espectro extendido que se recuperaron de urocultivos fueron conservados dentro de viales que contenían caldo tripticasa de soya con glicerol al $20 \%$ y mantenidos en refrigeración a-20 ㄷ hasta su posterior procesamiento. La extracción de plásmidos se realizó usando el kit GeneJET ${ }^{\mathrm{TM}}$ Plasmid Miniprep de Thermo Scientific Molecular Biology, que permitió obtener el ADN plasmídico en su forma circular. Se prepararon geles de agarosa al 0,8\% para colocar cada muestra en cada pocillo junto con el marcador de peso molecular de doble cadena de 1 Kb de GeneRuler $^{\mathrm{TM}}$ de Thermo Scientific - Molecular Biology, y realizar la corrida a un voltaje de $100 \mathrm{~V}$ durante 45 minutos, para luego visualizar los patrones de bandas obtenidos haciendo uso de un transiluminador ultravioleta Fotodyne 1.1430.

La información obtenida de las fichas de recolección de datos de los diferentes aislamientos de Escherichia coli y Klebsiella pneumoniae productoras de ß- lactamasas de espectro extendido, fueron ingresados en una base de datos, para lo cual se utilizó Microsoft Excel XP Profesional 2010. Para la elaboración de los dendrogramas se generó una matriz de presencia o ausencia de bandas, en base a los perfiles plasmídicos obtenidos en las corridas electroforéticas, representadas por 0 y 1 (0: ausencia y 1 : presencia) para lo cual se utilizó Microsoft Excel XP Profesional 2010 y se analizó mediante una matriz de similitud haciendo uso del software de análisis taxonómico multivariado NTSYS-PC versión 2.1; el cual calculó un coeficiente de similitud entre pares de conjuntos variables, transformando estos coeficientes en las distancias y haciendo un agrupamiento utilizando el algoritmo UPGMA. Se elaboraron dendrogramas (diagrama de grupos formado por conglomerados para establecer similitud) de los aislamientos de origen asociados a atención hospitalaria así como de origen comunitario, tanto de Escherichia coli y Klebsiella pneumoniae, y un dendrograma en conjunto de todos los aislamientos obtenidos para proceder al análisis de los mismos y de esta manera poder establecer la posible relación genética existente entre ellos. 


\section{RESULTADOS}

Se obtuvieron 56 aislamientos de Escherichia coli, a los cuales se les realizó pruebas de susceptibilidad para determinar su patrón frente a diferentes familias de antibióticos, así como la presencia de BLEE. Se determinó la resistencia del $100 \%$ de los aislamientos a la Cefotaxima (cefalosporina de 3 a generación), indicador de la presencia de BLEE en los aislamientos, así como la multirresistencia a otras familias de antibióticos en la mayoría de aislamientos. Figura 1.

De igual manera, se recuperó 6 aislamientos de Klebsilella pneumoniae, a los cuales se les realizó sus pruebas de susceptibilidad para determinar su patrón frente a diferentes familias de antibióticos, así como la presencia de BLEE. Se observó resistencia del $100 \%$ de los aislamientos a la Cefotaxima, Ceftazidima y Cefepime (cefalosporinas de $3 \underline{a}$ y $4^{a}$ ge- neración respectivamente), indicador de la presencia de BLEE en los aislamientos, así como la multirresistencia a otras familias de antibióticos en la mayoría de aislamientos, al igual que en los aislamientos de Escherichia coli.

Luego de la determinación de los perfiles plasmídicos, el plásmido más común que presentaron los aislamientos de Escherichia coli y Klebsiella pneu-

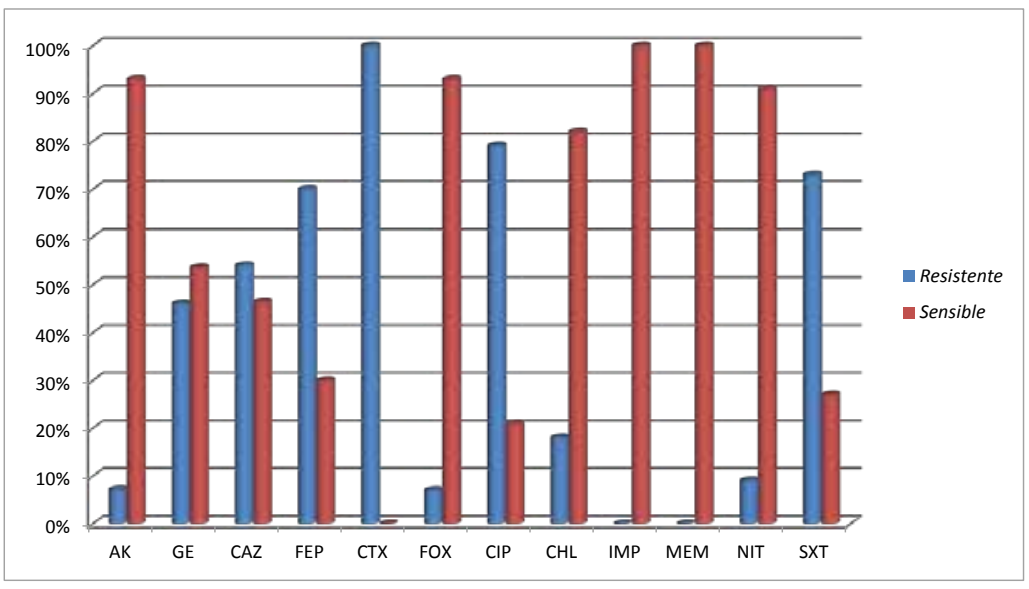

Figura 1. Patrón de susceptibilidad de Escherichia coli de urocultivos en el Instituto Nacional del Niño. Lima, Perú AK: Amikacina GE: Gentamicina CAZ: Ceftazidima FEP: Cefepime CTX: Cefotaxima FOX: Cefoxitina CIP: Ciprofloxacina CHL: Cloranfenicol IMP: Imipenem MEM: Meropenem NIT: Nitrofurantoína SXT: Trimetoprim-Sulfametoxazol.

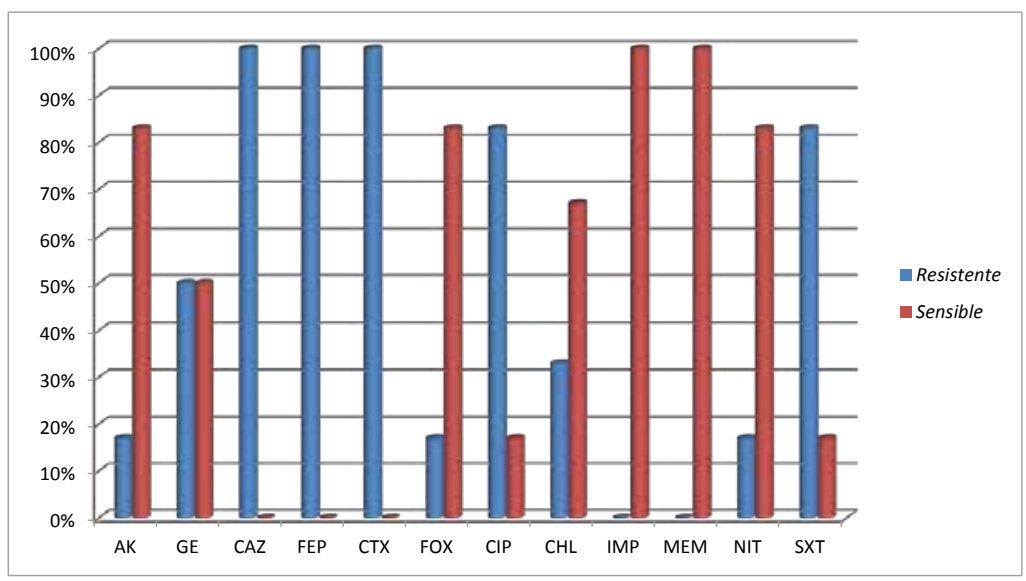

Figura 2. Patrón de susceptibilidad de Klebsiella pneumoniae de urocultivos de pacientes hospitalizados en el Instituto Nacional del Niño. Lima, Perú

AK: Amikacina GE: Gentamicina CAZ: Ceftazidima FEP: Cefepime CTX: Cefotaxima FOX: Cefoxitina CIP: Ciprofloxacina

CHL: Cloranfenicol IMP: Imipenem MEM: Meropenem NIT: Nitrofurantoína SXT: Trimetoprim-Sulfametoxazol. 
moniae fue el de $20 \mathrm{~Kb}(53 \%)$ como se aprecia en la figura 3. Los perfiles plasmídicos de los aislamientos de Escherichia coli mostraron de 1 a 7 bandas con tamaños entre aproximadamente $1.5 \mathrm{~Kb}$ hasta $20 \mathrm{~Kb}$, solo 3 aislamientos presentaron un plásmido con un tamaño mayor a $20 \mathrm{~Kb}$ (Figura 4); en el caso de los aislamientos de Klebsiella pneumoniae, pre- sentaron de 1 a 5 bandas con un tamaño molecular entre aproximadamente $2 \mathrm{~Kb}$ hasta $20 \mathrm{~Kb}$ y solo 1 aislamiento presentó un plásmido con un tamaño mayor a $20 \mathrm{~Kb}$.

En la figura 5 se observa el dendograma (diagrama de grupos formado por conglomerados para establecer simili-

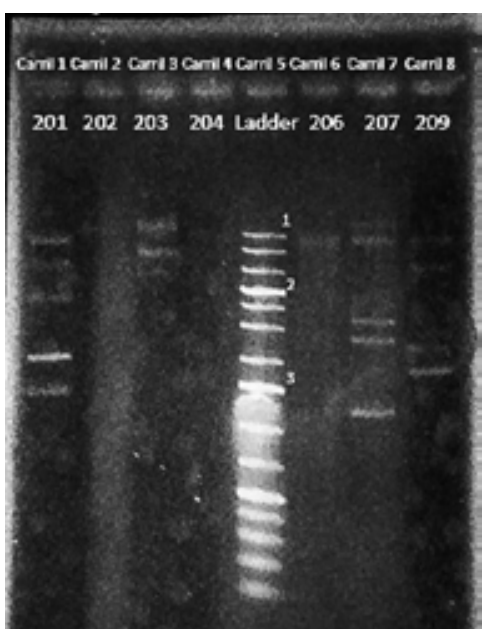

Figura 3. Perfiles plasmídicos de Escherichia coli y Klebsiella pneumoniae

Carril 1: Mx 201 (E. coli), carril 2: Mx 202 (E. coli), carril 3: Mx 203 (K. pneumoniae), carril 4: Mx 204 (E. coli), carril 5: Ladder: 1 Kb plus, carril 6: M× 206 (E. coli), carril 7: M× 207 (E. coli), carril 8: M× 209 (E. coli).
1: Ladder $20000 \mathrm{pb}$

2: Ladder $5000 \mathrm{pb}$

3: Ladder $1500 \mathrm{pb}$

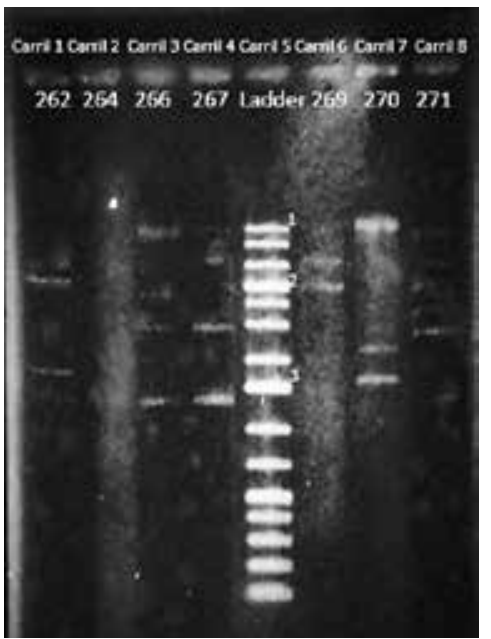

Figura 4. Perfiles plasmídicos de Escherichia coli
1: Ladder $20000 \mathrm{pb}$ 2: Ladder $5000 \mathrm{pb}$ 3: Ladder $1500 \mathrm{pb}$

Carril 1: Mx 262 (E. coli), carril 2: Mx 264 (E. coli), carril 3: Mx 266 (E. coli), carril 4: Mx 267 (E. coli), carril 5: Ladder: 1 Kb plus, carril 6: Mx 269 (E. coli), carril 7: Mx 270 (E. coli), carril 8: Mx 271 (E. coli)

tud) que muestra 24 genotipos distintos entre los aislamientos de Escherichia coli y Klebsiella pneumoniae tanto de origen asociados a atención hospitalaria como comunitario (línea vertical); 4 grupos formados presentaron aislamientos con el $100 \%$ de similitud genética, uno de ellos formado por 16 aislamientos de los cuales solo un aislamiento corresponde a Klebsiella pneumoniae (Mx: 218) y dos de ellos a aislamientos asociados a atención hospitalaria por Escherichia coli (Mx: 247, 254). De los otros 3 grupos con el $100 \%$ de similitud, 2 de ellos también incluyeron un aislamiento de Escherichia coli asociados a atención hospitalaria (Mx: 279, 321). En la figura 6 se aprecia el dendograma que incluye solo aislamientos de Escherichia coli de origen comunitario donde se observaron 18 genotipos donde el número de los grupos que presentaban el $100 \%$ de similitud genética se redujo a 3 . En la figura 7 se aprecia el dendrograma compuesto por los aislamientos de Escherichia coli de origen hospitalario, solo se observaron 7 genotipos distintos y tan solo un grupo con aislamientos con el $100 \%$ de similitud genética. En la figura 8 se muestra los dendogramas en los que se analizaron los aislamientos de Klebsiella pneumoniae de origen comunitario y asociados a atención hospitalaria respectivamente, la similitud genética que se observó fue del $0 \%$, demostrando que no existe relación alguna entre los aislamientos analizados.

Por tanto, al evaluar la relación entre los perfiles plasmídicos y los perfiles de susceptibilidad obtenidos, en el caso de los aislamientos de Escherichia coli, se puede determinar que se trata de cepas similares, pero no idénticas, excepto 5 aislamientos que presentaron el $100 \%$ de similitud de acuerdo al dendograma y tenían el mismo patrón de susceptibilidad antimicrobiana, por lo que se tratarían de una misma cepa. Los aislamientos de Klebsiella pneumoniae, luego de evaluar la relación entre los perfiles plasmídicos y los perfiles de susceptibilidad obtenidos, se tratarían de cepas totalmente distintas ya que no se observó relación alguna en los dendrogramas obtenidos, al presentar el $0 \%$ de similitud genética entre ellos. Así, no se encontró una relación significativa entre los perfi- 


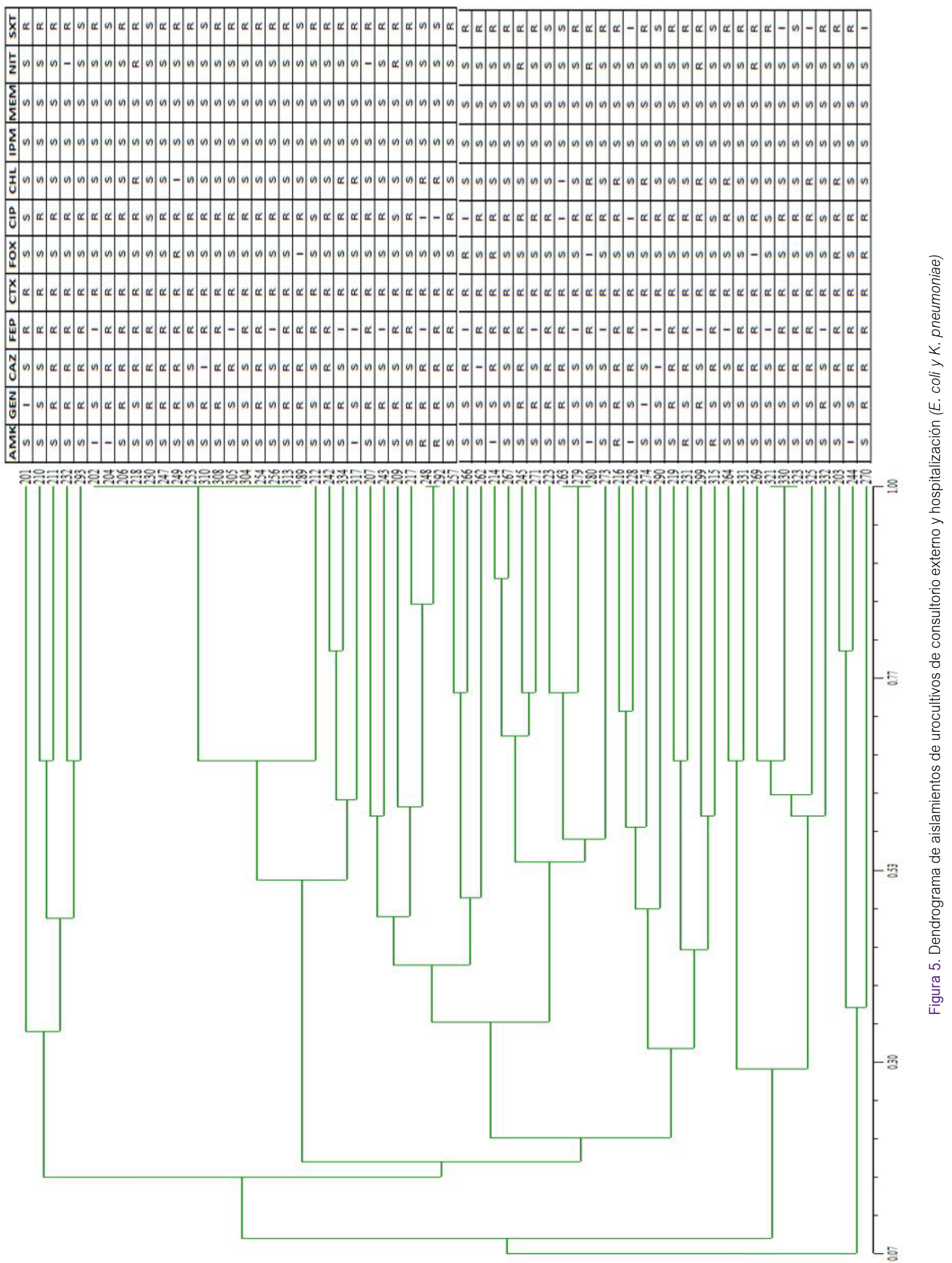




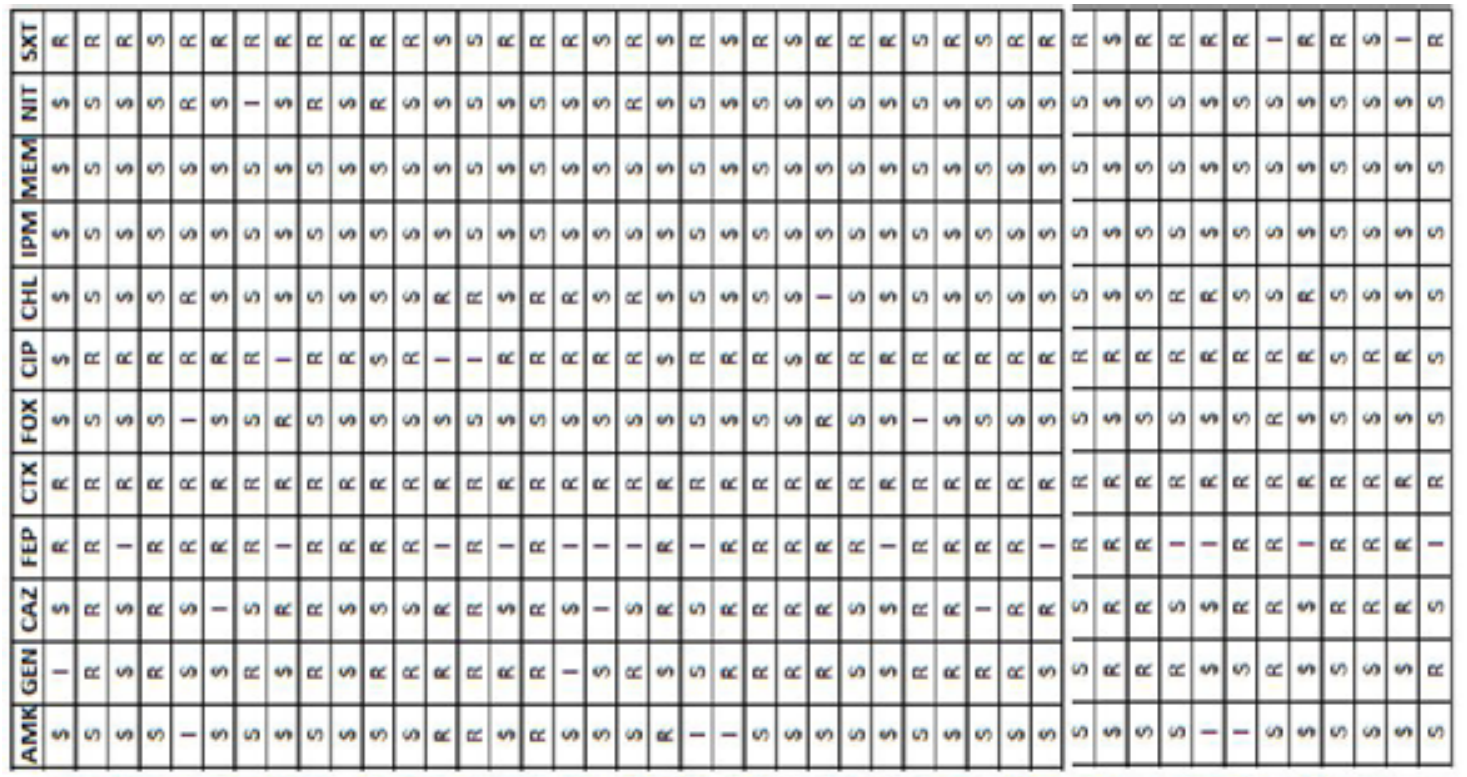

家高

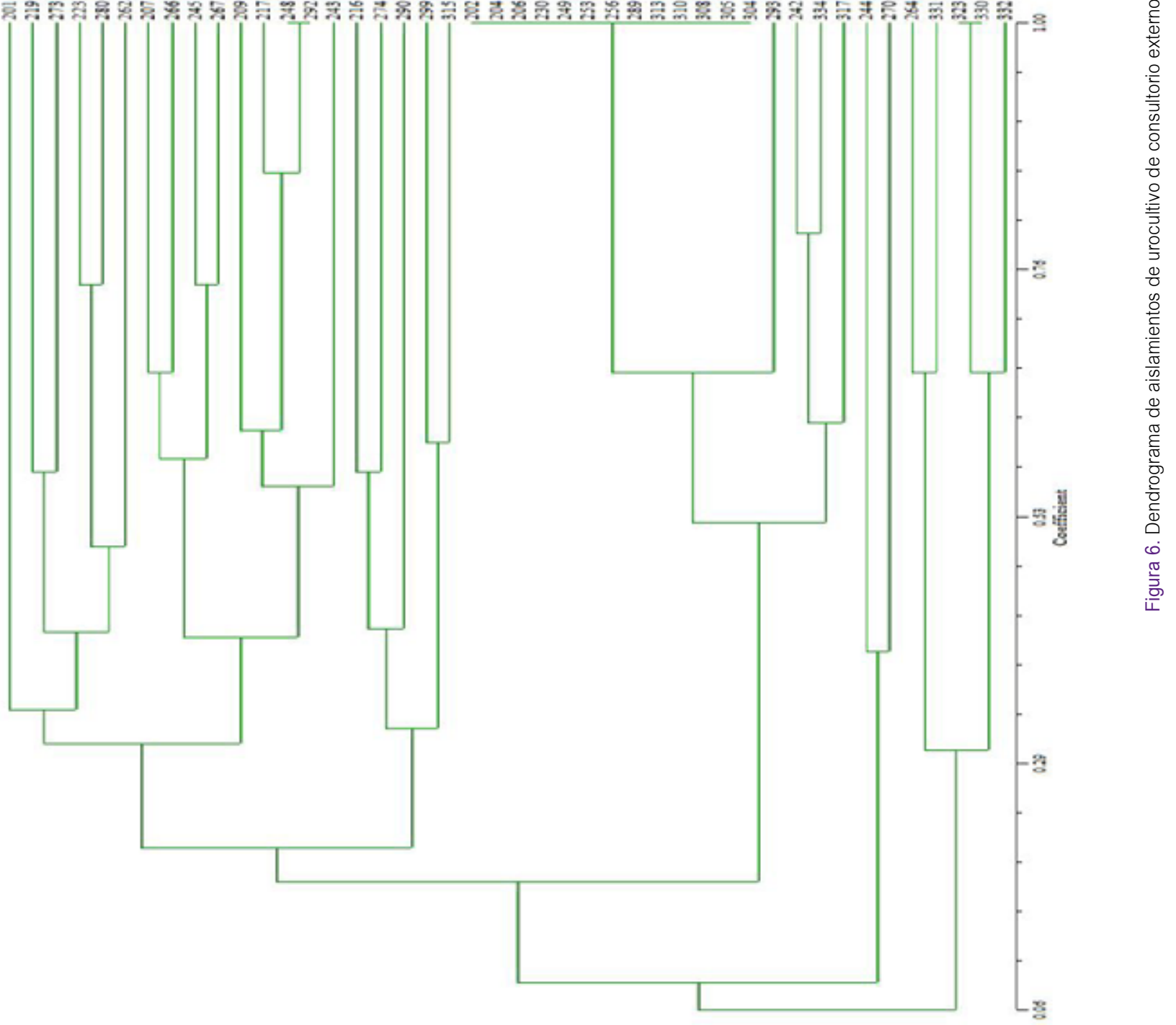



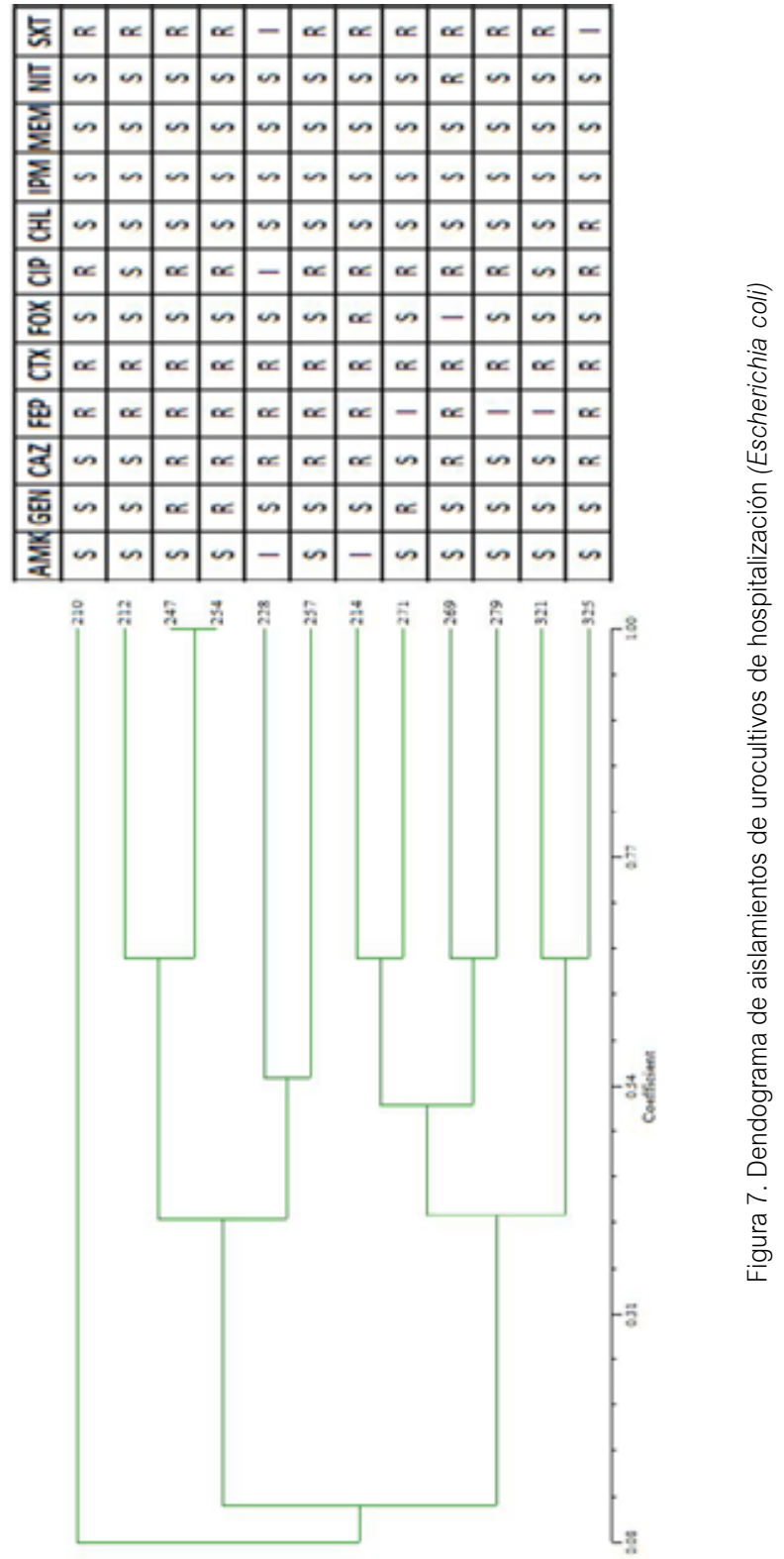
les plasmídicos obtenidos y los patrones de susceptibilidad antimicrobiana de los aislamientos estudiados por lo que no se pudo determinar un origen común entre los mismos.

\section{DISCUSIÓN}

Los patrones de susceptibilidad mostraron que los antibióticos que presentaron mayor resistencia, sin considerar a los $\beta$-lactámicos, fueron la ciprofloxacino (CIP) y el trimetoprim-sulfametoxazol (SXT) tanto en los aislamientos de Escherichia coli $(78,57$ y 73,21 \% respectivamente) como en los de Klebsiella pneumoniae (83,33 \% en ambos casos), resultados cercanos con los datos obtenidos en el estudio realizado por Marhova en Bulgaria, en el que el $71,43 \%$ de los aislamientos de Escherichia coli presentó resistencia al trimetoprim-sulfametoxazol, pero solo el 46,43\% a ciprofloxacino; asimismo, en un estudio realizado en la India con aislamientos de bacterias uropatógenas en pacientes pediátricos, el $70 \%$ de los aislamientos de Escherichia coli presentó resistencia al trimetoprimsulfametoxazol mientras que en Klebsiella pneumoniae fue del $50 \%{ }^{(9,10)}$.

La resistencia a 3 o más familias de antibióticos estuvo presente en el 77,5 $\%$ de los aislamientos. Este resultado es muy similar al del estudio realizado por S. Farshad y colaboradores en la India, con un porcentaje del $77 \%$ de los aislamientos con multidrogorresistencia; en Bangladesh, en el estudio de T. Lina el $87 \%$ de los aislamientos de Escherichia coli y Klebsiella pneumoniae presentaron resistencia a por lo menos 3 clases de antibióticos diferentes ${ }^{(11,12)}$.

Al presentar los aislamientos tanto de Escherichia coli y de Klebsiella pneumoniae el $100 \%$ de sensibilidad frente a los carbapenémicos, estos se convierten en la alternativa terapéutica contra microorganismos multirresistentes en infecciones del tracto urinario; resultado que concuerda con los obtenidos en los estudios de Farshad y Mustafa Aladog en Turquía, en los que se reportó también una alta sensibilidad a estos antibióticos

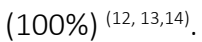

Luego de la determinación de los perfiles plasmídicos, el plásmido más común que presentaron los aislamientos de Escherichia coli y Klebsiella pneumoniae fue el de $20 \mathrm{~Kb}$ (53 \%), tamaño cercano al descrito por A. Celebi, en el que el plásmido de $19 \mathrm{~Kb}$ fue el más común en todos los aislamientos, S. Khadgi y colaboradores también describieron un plásmido de aproximadamente $23 \mathrm{~Kb}$ como el más común entre los aislamientos estudiados, y Nasreen, en la India, haciendo uso de una enzima de restricción encontraron como el plásmido más común el de 26 Kb $(15,16,17)$.

Los perfiles plasmídicos de los aislamientos de Escherichia coli mostraron de 1 a 7 bandas con tamaños entre aproximadamente $1.5 \mathrm{~Kb}$ hasta $20 \mathrm{~Kb}$, solo 3 aislamientos presentaron un plásmido con un tamaño mayor a $20 \mathrm{~Kb}$; en el caso de los aislamientos de Klebsiella pneumoniae presentaron de 1 a 5 bandas con un tamaño molecular entre aproximadamente $2 \mathrm{~Kb}$ hasta $20 \mathrm{~Kb}$ y solo 1 aislamiento presentó un plásmido con un tamaño mayor a $20 \mathrm{~Kb}$. En el estudio realizado por S. Farshad los aislamientos de Escherichia coli tenían entre 1 a 10 bandas con un rango de tamaños más amplio desde $1 \mathrm{~Kb}$ llegando hasta los $33 \mathrm{~Kb}$; en el estudio de Vásquez y colaboradores con aislamientos de Klebsiella pneumoniae, en el análisis de los perfiles plasmídicos de estos aislamientos reveló de 1 a 9 bandas distintas, mientras que Mustafa Aladog y colaboradores determinaron en los distintos aislamientos de su estudio productores de $ß$-lactamasas de espectro extendido de 1 a 8 bandas con tamaños entre $1.6 \mathrm{~Kb}$ y $30.1 \mathrm{~Kb}^{(12,13,18)}$.

Finalmente, no se encontró una relación significativa entre los perfiles plasmídicos obtenidos y los patrones de susceptibilidad antimicrobiana de los aislamientos estudiados mas que en tan solo 5 aislamientos, por lo tanto no se puede determinar un origen común entre los mismos; caso similar fue el estudio de M. Vasquez en el que no se encontró asociación entre los perfiles plasmídicos y las variables estudiadas. A. Celebi y col. tampoco encontraron una relación cercana entre los perfiles plasmídicos y la resistencia a los antibióticos de los aislamientos estudiados; asimismo, nin- guna relación pudo ser detectada entre el patrón de resistencia antimicrobiana y el análisis de los perfiles plasmídicos en el estudio llevado a cabo por S. Khadgi debido a las limitaciones de la técnica utilizada. Esto impulso la utilización de otros métodos de genotipificación en el estudio de brotes para demostrar la relación clonal entre aislamientos como en el estudio realizado por $\mathrm{H}$. Bailon y R. Sacsaquispe en Perú, en el que se hizo uso de tres métodos: ERIC-PCR, REP-PCR y electroforesis de campo pulsado (PFGE) pudiendo demostrar la relación clonal entre aislamientos de Klebsiella pneumoniae productores de ß-lactamasas de espectro extendido $(15,16,18,19)$.

Se debe realizar cada paso de la extracción de ADN plasmídico con mucho cuidado y respetando las indicaciones establecidas en el protocolo de extracción, ya sea usando un estuche comercial o la técnica de lisis alcalina propuesta por Birnboim y Doly ${ }^{(20)}$ para de esta manera asegurar la mayor cantidad de ADN extraído y una óptima calidad del mismo, debido a la importancia de este tipo de estudios ya que el ADN plasmídico juega un papel muy importante en la transmisión de este tipo de resistencia como las BLEE, ya que nos permite obtener datos claves en el control de las mismas.

Como limitaciones del estudio mencionamos que otra técnica molecular como la reacción en cadena de la polimerasa podría confirmar el tipo de ß-lactamasa de espectro extendido presente en cada aislamiento estudiado y con ello tener mayor información que nos facilite el análisis con respecto a los perfiles plasmídicos obtenidos, ya que los métodos de susceptibilidad son métodos fenotípicos y dependen de muchos factores como inóculo bacteriano, lectura del analista, etc. Por el contrario, los métodos moleculares son más sensibles y específicos por lo que algunas veces no concuerdan los resultados; y al ser la determinación de perfiles plasmídicos una de las técnicas más sencillas para el análisis de relación clonal entre aislamientos bacterianos, no se obtuvieron mejores resultados a los que se podrían obtener si se hubiera usado otra técnica de mayor complejidad que nos permitiera encontrar un origen común entre los asilamientos. 
Concluimos que luego de la determinación de los perfiles plasmídicos, el plásmido con un tamaño de $20 \mathrm{~Kb}$ fue el más común, presente en el $53 \%$ de los aislamientos, de los cuales el $76 \%$ fue resistente a ciprofloxacina y el $73 \%$ a trimetoprim-sulfametoxazol, pudiendo ser uno de los plásmidos responsables de la transmisión de las ß-lactamasas de espectro extendido y la resistencia a estos antibióticos en particular, pero se necesitarían de mayores estudios para poder confirmar esto. Luego de evaluar la relación entre los perfiles plasmídicos y los perfiles de susceptibilidad obtenidos, en el caso de los aislamientos de Escherichia coli, se puede determinar que se trata de cepas similares, pero no idénticas, excepto 5 aislamientos que presentaron el 100 $\%$ de similitud de acuerdo al dendograma y tenían el mismo patrón de susceptibilidad antimicrobiana, por lo que se tratarían de una misma cepa. Los aislamientos de Klebsiella pneumoniae, luego de evaluar la relación entre los perfiles plasmídicos y los perfiles de susceptibilidad obtenidos, se tratarían de cepas totalmente distintas ya que no se observó relación alguna en los dendrogramas obtenidos, al presentar el $0 \%$ de similitud genética entre ellos. Al presentar los aislamientos tanto de Escherchia coli como de klebsieIla pneumoniae el $100 \%$ de sensibilidad frente a los carbapenémicos, se convierten en la alternativa terapéutica contra estos microorganismos multirresistentes, por lo que es importante que estos agentes se usen con moderación y discreción; además de la monitorización continua de estos antimicrobianos con respecto a su efectividad. El estudio adicional a nivel molecular de los aislamientos con otras técnicas puede ser beneficioso para establecer la causa del patrón multidrogorresistente que puede ayudar a hacer una contribución a la comprensión actual y al conocimiento de la situación de la infecciones del tracto urinario de origen bacteriano causada por agentes patóge- nos multidrogorresistentes, y para el desarrollo de una mejor estrategia de tratamiento y prevención de la enfermedad.

\section{AGRADECIMIENTOS}

Al Centro de Investigación de Bioquímica y Nutrición "Alberto Guzmán Barrón" de la Facultad de Medicina de la Universidad Nacional Mayor de San Marcos y al Instituto Nacional de Salud del Niño por su apoyo en la investigación.

\section{REFERENCIAS BIBLIOGRÁFICAS}

1. Organización Mundial de la Salud. Movilizar la voluntad política para contener la resistencia a los antimicrobianos. Bulletin of the World Health Organization. 2011; 89:168-169.

2. Livermore DM. Current epidemiology and growing resistance of gram negative pathogens. Korean J Intern Med. 2012; 27: 128-142. DOI: 10.3904/ kjim.2012.27.2.128

3. Bush K., Jacoby G. Updated Functional Classification of $\beta$-Lactamases. Antimicrob Agents Chemother. 2010; 54(3): 969-976. DOI: 10.1128/ AAC.01009-09

4. Perozo M, Armindo J, Castellano G, Maribel J. Detección de $\beta$-lactamasas de Espectro Extendido en cepas de la familia Enterobacteriaceae. Kasmera. 2009; 37(1):25-37.

5. Díaz M, Ramón J, Martínez L, Rodríguez J, Pascual A, Grupo de estudio de infección hospitalaria (GEIH). Escherichia coli y Klebsiella pneumoniae productoras de $\beta$-lactamasas de espectro extendido en hospitales españoles: segundo estudio multicéntrico (proyecto GEIH-BLEE 2006). Enferm Infecc Microbiol CIIN. 2009; 27(9):503-510. DOI: 10.1016/j.eimc.2008.09.006

6. Alvarez E, Zayas A, Castillo I, Gonzales L, Contreras R. Detección de aislados clínicos de Escherichia coli y Klebsiella spp. productoras de $\beta$-lactamasas de espectro extendido mediante el sistema DIRAMIC. Revista CENIC Ciencias Biológicas. 2010; 41(3):195-199.

7. Martinez L. Asociación de BLEE con otros mecanismos de resistencia. Enferm Infecc Microbiol Clin. 2007; 25(Supl. 2): 38-47.

8. Navarro F, Miro E. Entorno genético de las BLEE: implicaciones en la transmisión. Enfer Infecc Microbiol Clin. 2007; 25(Supl. 2):11-7.

9. Marhova M, Kostadinova S, Stoitsova S. Antimicrobia resistance profiles of urinary Escherichia coli isolates. Biotechnol. \& Biotechnol. EQ. 2009; 23: 616-620. DOI: https://doi.org/10.1080/13102818.2009.10818500
10. Ranjbar R, Haghi-Ashtiani MT, Jonaidi Jafari N, Abedini $\mathrm{M}$. The prevalence and antimicrobial susceptibility of bacterial uropathogens isolated from pediatric patients. Iranian J Publ Health. 2009; 38(2):134-138.

11. Lina TT, Rahman SR, Gomes DJ. Multiple-Antibiotic Resistance Mediated by Plasmids and Integrons in Uropathogenic Escherichia coli and Klebsiella pneumoniae. Bangladesh J Microbiol. 2007; 24 (1): 19-23

12. Farshad S, Ranjbar R, Japoni A, Hosseini M, Anvarinejad M, Mohammadzadegan R. Microbial Susceptibility, Virulence Factors, and Plasmid Profiles of Uropathogenic Escherichia coli strains isolated from children in Jahrom, Iran. Arch Iran Med. 2012; 15 (5): 312 - 316. DOI: 012155/AIM.0013.

13. Mustafa OA, Yusuf D, Ahmet U. Investigation Of Imipenem and Meropenem susceptibilities, Plasmid Profiles and ESBL characteristic of Klebsiella neumonia isolated from urinary tract infections. World Applied Sciences Journal. 2009; 7 (3): 378-381.

14. Giedraitiené A, Vitkauskiené A, Naginiené R, Pavilonis A. Antibiotic resistance mechanisms of clinically important bacteria. Medicina (kaunas). 2011; 47(3):137- 146

15. Çelebi A, Duran N, Öztürk F, Açik L, Aslan G, Aslantas O. Identification of clinic uropathogen Escherichia coli isolates by antibiotic susceptibility, plasmid and whole cell protein profiles. Advances in Molecular Biology. 2007; (1): 31-40.

16. Khadgi S, Timilsina U, Shrestha B. Plasmid profiling of multidrug resistant Escherichia coli strains isolated from urinary tract infection patients. Int J Appl Sci Biotechnol. 2013; 1(1): 1-4. DOI: http:// dx.doi.org/10.3126/ijasbt.v1i1.7918

17. Nasreen J, Sudhir U, Meshram, Archana K. Plasmid profile analysis of multidrug resistant E. coli isolated from UTI patients of Nagpur City, India. Romanian Society of Biological Sciences. 2009; 14 (5): 4635-4640.

18. Vásquez M, Moreno N, Correa M, Estrada J, Castañeda L. Caracterización bioquímica, patrón de sensibilidad y perfil plasmídico de cepas hospitalarias multirresistentes de Klebsiella pneumoniae. IATREIA. 2001; 14 (4):291.

19. Bailón H, Sacsaquispe R. Caracterización molecular de cepas de Klebsiella pneumoniae productoras de BLEE causantes de infección intrahospitalaria en el servicio de neonatologia de un hospital de Lima, Perú. Rev Med Hered. 2013; 24:101-108.

20. Birnboim HC, Doly J. A rapid alkaline extraction procedure for screening recombinant plasmid DNA. Nucleic Acids Res. 1979; 7: 1513-1523. 